\title{
A multi-scale friction model framework for full scale sheet forming simulations
}

\author{
J. Hol*, T. Meinders ${ }^{\dagger}$ and J. Huétink ${ }^{\dagger}$ \\ ${ }^{*}$ Materials innovation institute (M2i), P.O. box 5008, 2600 GA Delft, the Netherlands \\ ${ }^{\dagger}$ University of Twente, Faculty of Engineering Technology, chair of Forming Technology, P.O. box 217, 7500 AE \\ Enschede, the Netherlands
}

\begin{abstract}
In this paper a numerical framework is proposed which accounts for the most important friction mechanisms. Static flattening and flattening due to bulk strain are accounted for by theoretical models on a microscale. Based on statistical parameters a fast and efficient translation from micro- to macro modeling is included. A general overview of the friction model is presented and the translation from micro to macro modeling is outlined. The development of real area of contact is described by the flattening models and the effect of ploughing and adhesion on the coefficient of friction is described by a micro-scale friction model. A brief theoretical background of these models is given. The flattening models are validated by means of FE simulations on microscale and the feasibility of the advanced macroscopic friction model is proven by a full scale sheet metal forming simulation.
\end{abstract}

Keywords: friction, ploughing, adhesion, flattening, roughening, coefficient of friction

PACS: 46.15.-x, 46.35.+z, 46.55.+d

\section{INTRODUCTION}

The automotive industry relies on finite element simulations to predict formability of newly designed car body components. The usability of such a code largely depends on the accuracy of the numerical models in these codes. An accurate forming analysis can only be made if, amongst others, the friction conditions are modeled accurately. However, in the majority of simulations still a simple Coulomb friction model is used. The Coulomb friction model assumes a constant friction coefficient which is independent of parameters such as pressure, punch speed or deformation of the sheet material. However, in reality, the friction coefficient does depend on these parameters. Hence, it is still cumbersome to predict the material flow during large scale forming processes correctly.

In the past, research has been carried out on contact and friction phenomena on different length scales. This paper focuses on the phenomena which play a role in the boundary lubrication regime, which is the most common condition during sheet metal forming. Ploughing effects between asperities and adhesion effects between boundary layers are the main factors causing friction in the boundary lubrication regime. On the microscopic level, friction is due to adhesion between contacting asperities [1,2] and the ploughing effect between asperities [1,2]. Wilson [1] developed a model which treated the effect of adhesion and ploughing separately. A more advanced model was developed by Challen \& Oxley [2] which takes the combined effect of ploughing and adhesion on the coefficient of friction into account. Westeneng [3] extended this model to describe friction conditions between multiple tool asperities and a flat workpiece material.

The friction coefficient depends on the amount of ploughing and adhesion, which depends on the real area of contact. The real area of contact is defined by the roughness of both tool and workpiece, where the roughness of the workpiece is liable to changes due to flattening and roughening mechanisms. The main flattening mechanisms during sheet metal forming are flattening due to normal loading [4], flattening due to stretching [5, 6] and flattening due to sliding [7]. Flattening increases the real area of contact, resulting in a higher coefficient of friction. The two mechanisms outlined in this paper are flattening due to normal loading and flattening due to stretching. Future work is planned on modeling the roughening effect and the influence of sliding on the flattening behavior.

Most models to predict the flattening behavior of asperities due to normal loading are based on the work of Greenwood \& Williamson [4] which developed a stochastic model based on contact between a flat tool and rough workpiece surface. During the last decades, modifications have been made to this model to account for arbitrary shaped asperities, plastically deforming asperities and the interaction between asperities [8],[9], [10], [11], [12]. The model of Pullen \& Williamson inspired Westeneng [3] to derive an ideal-plastic and nonlinear-plastic contact model 
based on the conservation of volume and energy. Westeneng modeled the asperities as bars which can represent arbitrarily shaped asperities. The model includes work-hardening and is able to describe the interaction between asperities.

Bulk strain can have an accelerating or decelerating effect on the change in real area of contact. The effective hardness of asperities will reduce if a positive bulk strain is present in the underlying material [5]. Wilson \& Sheu [5] developed a semi-empirical plane stress model to describe the flattening behavior using wedge-shaped asperities with a constant angle. The model provides a relation between the effective hardness, the real area of contact and a non-dimensional strain rate. Sutcliffe [6] extended the model of Wilson \& Sheu to describe a plane-strain situation in the direction of the asperities based on slip line theory. Westeneng [3] developed a strain model which describes the influence of strain on a surface geometry using arbitrary shaped asperities. This versatile model is applicable to both plane-strain and plane-stress situations, depending on the definition of the non-dimensional strain rate.

In this paper, a friction model is proposed which includes flattening due to normal loading and flattening due to straining, ploughing and adhesion. The contact models of Westeneng are used to describe the flattening behavior of asperities due to normal loading and due to stretching under normal loading. The influence of ploughing and adhesion on the coefficient of friction has been described by the extended model of Challen \& Oxley. A brief overview of the friction model is presented and the translation from micro to macro modeling is outlined. The theoretical background of the models used to describe the various friction mechanisms are briefly discussed in Section 'Theory'. In Section 'Validation' the flattening models are validated by means of finite element simulations on the micro-scale. Finally, Section Macro-scale deep drawing simulation' shows the feasibility of the advanced macroscopic friction model by a full scale sheet metal forming simulation.

\section{THEORY}

The real area of contact is calculated based on the models accounting for flattening due to normal loading and flattening due to stretching. The amount of indentation of the harder tool asperities into the softer workpiece asperities can be calculated if the real area of contact and the contact pressure carried by the asperities are known. After that, shear stresses due to ploughing and adhesion effects between asperities and the coefficient of friction are calculated. In this section a brief explanation of the theory will be provided. For a detailed derivation of the following equations, the reader is referred to [13]

\section{Flattening models}

Micro-scale friction models are too cumbersome to be used in large-scale FE simulations. Therefore, the contact behavior on micro-scale has to be translated to macroscopic contact behavior. The surface of tools and workpiece can be described by stochastic methods on the micro-scale. Statistical parameters can be used under the assumption that the surface texture is isotropic and can be represented by 2-dimensional correlated random noise. Assuming that the surface height distribution on the micro-scale represents the surface texture on the macro-scale, it is possible to describe contact problems that occur during large-scale FE analyzes of sheet metal forming processes [3].

A discrete surface height distribution of the tool and workpiece material is obtained from surface profiles. However, a continuous function is desirable to eliminate the need for integrating discrete functions during the solution procedure of the friction model. Therefore, a half range sine Fourier function [14] is used to fit the surface height distribution $\phi(z)$ :

$$
\phi(z)=\frac{2}{L} \sum_{n=1}^{\infty}\left[\int_{0}^{L} f(z) \sin \left(\frac{n \pi}{L} z\right) \mathrm{d} z\right] \sin \left(\frac{n \pi}{L} z\right)
$$

with $L$ the evaluation domain and $f(z)$ the discrete form of the surface height distribution.

The ratio of the real to the nominal contact area $\alpha$ is defined as:

$$
\alpha=\int_{d-U}^{\infty} \phi(z) \mathrm{d} z
$$


with $d$ the amount of flattening of the contacting asperities and $U$ the rise of the non-contacting asperities. The parameters $d$ and $U$ are calculated by simultaneously solving Equations 2, 3 and 4:

$$
\begin{aligned}
& \frac{P_{\text {nom }}}{H}=\xi\left(1+\eta \chi \int_{d}^{\infty} \phi(z) \mathrm{d} z\right) \\
& U(1-\alpha)=\int_{d-U}^{\infty}(z-d) \phi(z) \mathrm{d} z
\end{aligned}
$$

with $P_{\text {nom }}$ the nominal contact pressure (input parameter) and $H$ the hardness. $\xi$ can be regarded as an energy factor and $\chi$ as a shape factor. The persistence parameter $\eta$ describes the amount of energy required to lift up the non-contacting asperities. A value of $\eta=0$ means that no energy is needed to rise the asperities, a value of $\eta=1$ implies that a maximum amount of energy is needed to rise the asperities.

The above equations are based on a normal loading case without additional bulk strain. To account for flattening due to stretching, the models have to be adapted. The change of the fraction of the real contact area as a function of the nominal strain can be presented as:

$$
\frac{\mathrm{d} \alpha_{S}^{n}}{\mathrm{~d} \varepsilon}=\frac{l}{E} \phi\left(d_{S}^{n-1}-U_{S}^{n-1}\right)
$$

with $n$ the iteration number. The subscript $S$ is used for variables that become strain dependent. The contact area ratio is updated incrementally by:

$$
\alpha_{S}^{n}=\alpha_{S}^{n-1}+\mathrm{d} \alpha_{S}^{n}
$$

The initial values $\alpha_{S}^{0}, d_{S}^{0}$ and $U_{S}^{0}$ are obtained from the model without bulk strain. To calculate the change of $\alpha_{S}$, the value of $U_{S}$ and $d_{S}$ needs to be solved simultaneously while $\varepsilon$ is incrementally increased. Based on volume conservation (Equation 8) and the definition of the fraction of real contact area (Equation 7) $U_{S}$ and $d_{S}$ can be obtained.

$$
\begin{gathered}
\alpha_{S}=\int_{d_{S}-U_{S}}^{\infty} \phi(z) d z \\
U_{S}\left(1-\alpha_{S}\right)=\int_{d_{S}-U_{S}}^{\infty}\left(z-d_{S}\right) \phi(z) d z
\end{gathered}
$$

The equations in this section are derived under the assumption of elastic-ideal plastic material behavior. Recently the model for flattening due to normal contact only is extended for Nadai hardening. The equations belonging to this extended theory will be published in an upcoming paper. In this paper only the results of this extended model will be discussed.

\section{Friction coefficient}

Once the real area of contact is calculated with the above mentioned flattening models, the model of Westeneng is used to calculate the coefficient of friction between multiple hard asperity and a soft flat surface [15]:

$$
F_{w}=\rho_{t} \alpha_{S} A_{\text {nom }} \int_{\delta}^{s_{\max }} \mu_{a s p} \pi \omega \beta_{t} H \phi_{t}(s) d s
$$

with $\omega$ the amount of indentation and $\beta_{t}$ the mean radius of the tool asperities. $\rho_{t}$ represents the asperity density of the tool surface, $A_{n o m}$ the nominal contact area and $\phi_{t}$ the normalized surface height distribution function of the tool surface. The bounds of the integral are described by $s_{\max }$, the maximum height of the tool asperities, and $\delta$, the separation between the workpiece surface and the mean plane of the tool asperities. Since the normal force is known (input parameter), the coefficient of friction can finally be obtained by:

$$
\mu=\frac{F_{w}}{F_{N}}
$$


TABLE 1. Material and roughness parameters

\begin{tabular}{|c|c|c|c|c|c|}
\hline \multirow{2}{*}{ Material parameter } & \multirow{2}{*}{ Value } & \multirow{2}{*}{ Unit } & Roughness parameter & Value & Unit \\
\hline & & & & & \\
\hline & & & Hardness workpiece $(H)$ & 1400 & $\mathrm{MPa}$ \\
\hline Elastic modulus $(E)$ & 210 & $\mathrm{GPa}$ & Persistence parameter $(\eta)$ & 1 & \\
\hline Poisson's ratio $(v)$ & 0.3 & & Density workp. asp. $\left(\rho_{\text {work }}\right)$ & $5.0 \cdot 10^{3}$ & $\mathrm{~mm}^{-2}$ \\
\hline Initial strain $\left(\varepsilon_{0}\right)$ & 0.00243 & & Density tool. asp. $\left(\rho_{\text {tool }}\right)$ & $2.0 \cdot 10^{3}$ & $\mathrm{~mm}^{-2}$ \\
\hline$C$-parameter $(C)$ & 500 & $\mathrm{MPa}$ & Radius tool. asp. $\left(\beta_{t}\right)$ & $2.0 \cdot 10^{-2}$ & $\mathrm{~mm}$ \\
\hline$n$-parameter $(n)$ & 0.2 & & Nr. of Fourier expansions & & \\
\hline & & & Non-dim. strain rate $(E)$ & Sutcliffe & \\
\hline
\end{tabular}

\section{VALIDATION}

FE simulations on the micro-scale have been performed in order to validate the flattening models. In the simulations, a $4 \mathrm{~mm}$ long surface was indented by a perfectly flat and rigid tool. The roughness distribution used in the FE simulation equals the distribution measured for DC04 low-carbon steel. The bulk material was modeled by 4-node 2D plane-strain elements. The yield behavior was described by the Von Mises yield criterion and a Nadai hardening law. Material parameters used for the FE simulation are listed in Table 1. Contact between the tool and the rough surface was described by the penalty method. The surface height distribution used for the analytical model corresponds to the roughness distribution of the FE simulation. A fixed hardness of $450 \mathrm{MPa}\left(3 \sigma_{y}\right)$ was used in the analytical model since a yield strength of 150MPa was used for the FE simulation.

The analytical solution and the FE solution for elastic ideal-plastic and elastic non-linear plastic material behavior are presented in Figure 1 for flattening due to normal loading only. The material behavior of the elastic nonlinear plastic FE simulation is comparable to the material behavior assumed in the analytical model. The $R_{q}$ value represents the maximum peak to value distance. The analytical solution with $R_{q}=1$ corresponds reasonably well with the FE results. A perfect fit can be obtained by using $R_{q}$ as a fitting parameter $\left(R_{q}=2\right)$. The latter value is chosen in the remainder of this paper.

Combined normal loading and stretching the underlying bulk material decreases the effective hardness [5]. A lower hardness results in an increase of the real area of contact. Both the analytical and the FE results of this analysis ( rough surface has been indented by a nominal load while a bulk strain has been applied to the underlying material), are presented in Figure 1. It can be concluded that work-hardening effects have a large influence on the flattening behavior of the asperities. A difference of $20 \%$ in the real area of contact is obtained at the end of the simulation between the results of the elastic ideal-plastic and the elastic nonlinear-plastic simulation. The density of workpiece asperities (in $\mathrm{mm}^{-2}$ ) is an unknown parameter in the analytical strain model. Various methodologies exist to determine the asperity density from the surface profile. Results obtained by these methodologies are highly dependent on the chosen method and the resolution of the used roughness measurement device. Future work is planned to determine this parameter using the most suitable method. Until then, the asperity density will be taken as an unknown parameter. Calculations have been performed using realistic values for the asperity density for DC04 to show the importance of this parameter, see Figure 1. It can be concluded that the asperity density of the workpiece has a significant influence on the development of the real area of contact. The amount of indentation of the workpiece asperities will be lower if a higher value of the asperity density is used. Consequently, a lower amount of indentation results in a lower amount of real area of contact. The trend of the graphs corresponds well to the flattening behavior obtained by the FE simulations. Using an asperity density of $5000 \mathrm{asp} / \mathrm{mm}^{2}$ it is possible to describe the results of the elastic ideal-plastic FE solution precisely.

\section{MACRO-SCALE DEEP DRAWING SIMULATION}

A cross-die product is used to test the numerical performance of the developed friction model in a large-scale FE simulation. Due to symmetry only a quarter of the workpiece was modeled. The workpiece was meshed with 9000 triangular discrete Kirchhoff shell elements using 3 integration points in plane and 5 integration points in thickness direction. The material used is a DC04 low carbon steel. The yield behavior was described by the Vegter model [16] using the Bergström-Van Liempt hardening relation [17]. A penalty method was used to describe contact between the tools and the workpiece, using a penalty stiffness of $200 \mathrm{~N} / \mathrm{mm}$. The coefficient of friction used in the contact algorithm was calculated on the basis of the friction model presented in this paper. The simulation was performed by 

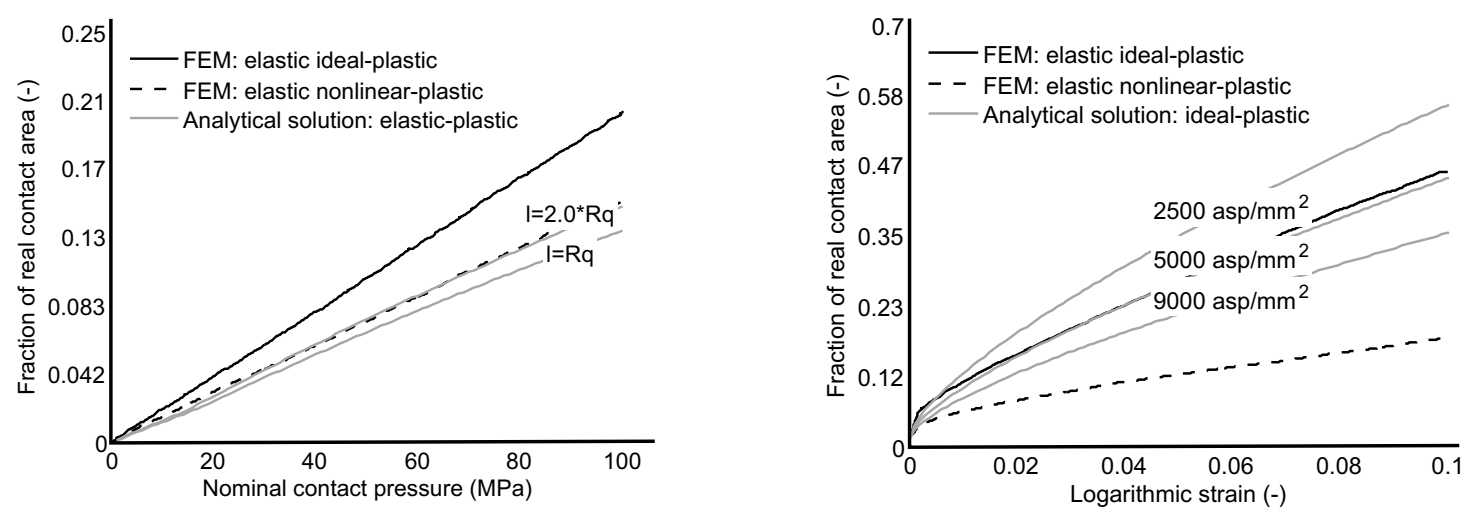

FIGURE 1. Fraction of real area of contact for normal loading (left) and normal loading + stretching (right)

prescribing the displacement until a punch stroke of $60 \mathrm{~mm}$. The punch speed was set to $5 \mathrm{~cm} / \mathrm{sec}$ and the blankholder force was set to $60 \mathrm{kN}$.
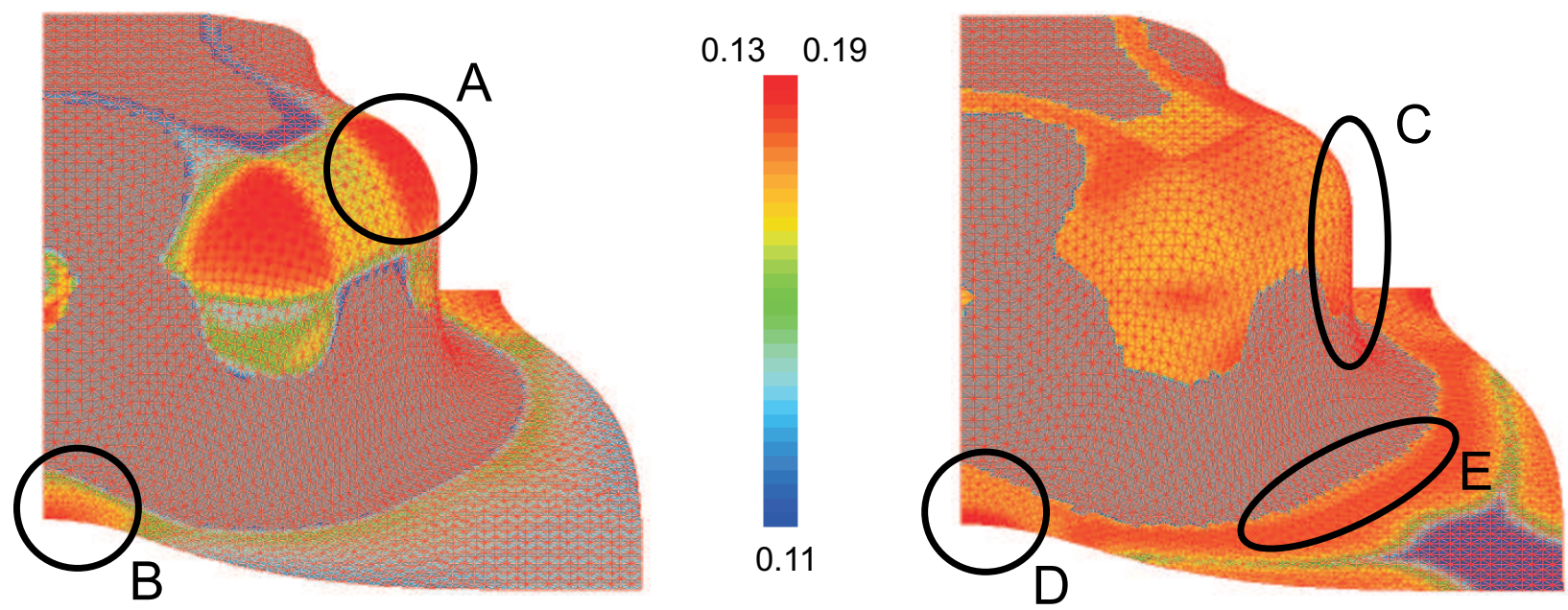

FIGURE 2. Friction coefficient for normal loading (left) and normal loading + stretching (right)

Two simulations have been performed in order to quantify the individual contributions of the two flattening mechanisms. The first simulation only accounted for the influence of normal loading on the coefficient of friction, Figure 2, left. The second simulation uses both flattening models to determine the coefficient of friction, Figure 2, right. Results shown are from the punch side of the sheet. The gray areas represent the non-contacting areas.

If only flattening due to static loading is assumed, rather low values for the ratio of the real to the nominal area of contact are obtained. This results in friction coefficients that vary between 0.11 and 0.13 . The higher values are obtained in high-pressure regions: the contact area of the punch radius (region A) and the thickened area in the blankholder region (region B). If the second flattening mechanism is taken into account (flattening due to stretching), significant higher values for the real area of contact are obtained. The higher contact ratios result in higher values of the coefficient of friction, i.e. between 0.11 and 0.19. It can be observed from Figure 2 (right) that higher values of the coefficient of friction occur at regions where high strains occur (region C, D and E). Region C is purely stretched, region $\mathrm{D}$ is compressed which causes thickening of the material and region $\mathrm{E}$ is stretched over the die radius. Overall it can be concluded that the distribution of the coefficients of friction lies within the range of expectation.

Finally, the draw-in patterns of both simulations are compared to the simulation results in which the standard Coulomb friction model is used with a friction coefficient of 0.11 . It can be observed that the draw-in in case of flattening due to normal loading only more or less equals the draw-in of the Coulomb friction model. This is logical since the friction coefficient distribution in case of normal loading only does not deviate that much from the value 0.11 . However, when stretching is also taken into account, the draw-in significantly deviates from the draw-in obtained with the Coulomb 
friction model. Again, this is logical since the maximum obtained friction coefficient in case of stretching is much higher than the fixed value of 0.11 .

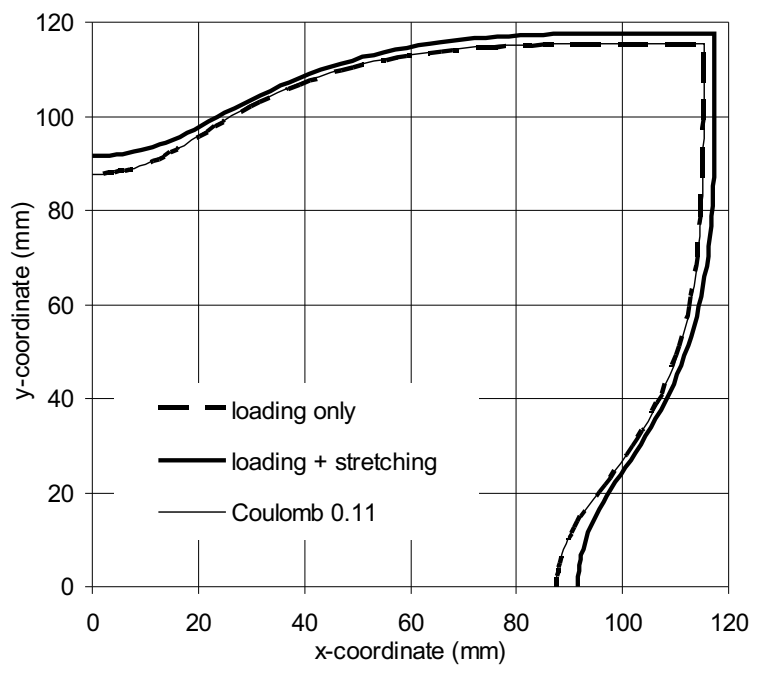

FIGURE 3. Flange draw-in for simulations with the Coulomb friction model $(\mu=0.11)$ and the both flattening models

In this paper, the focus is on the numerical performance and feasibility of the friction model that has been developed. The results look very promising. To validate this model, an experimental test procedure is currently being setup.

\section{ACKNOWLEDGMENTS}

This research was carried out under the project number MC1.07289 in the framework of the Research Program of the Materials innovation institute M2i (www.m2i.nl).

\section{REFERENCES}

1. W. Wilson, Americal Society of Mechanical Engineers 10, 13-23 (1988).

2. J. Challen, and P. Oxley, Wear 53, 229-243 (1979).

3. J. Westeneng, Modelling of contact and friction in deep drawing processes, Ph.D. thesis, University of Twente (2001).

4. J. Greenwood, and J. Williamson, Proceedings of the Royal Society of London. Series A, Mathematical and Physical sciences 295, 300-319 (1966).

5. W. Wilson, and S. Sheu, International Journal of Mechanical Science 30, 475-489 (1988).

6. M. Sutcliffe, International Journal of Mechanical Science 30, 847-868 (1988).

7. S. Lo, and T. Yang, Journal of Tribology 125, 713-719 (2003).

8. Y. Zhao, and L. Chang, Journal of Tribology 123, 857-864 (2001).

9. D. M. Y. Zhao, and L. Chang, Journal of Tribology 122, 86-93 (2000).

10. Y. Jeng, and P. Wang, Journal of Tribology 125, 232-240 (2003).

11. G. Pugliese, S. Tavares, E. Ciulli, and L. Ferreira, Wear 264, 1116-1128 (2008).

12. J. Pullen, and J. Williamson, Proceedings of the Royal Society of London. Series A, Mathematical and Physical sciences 327, 159-173 (1972).

13. J. Hol, M. C. Alfaro, M. de Rooij, and T. Meinders, WEAR (submitted in 2010).

14. M. Greenberg, Advanced Engineering Mathematics - 2nd edition, Prentice Hall, 1998.

15. J. Challen, and P. Oxley, International Journal of Mechanical Siences 26, 403-418 (1984).

16. H. Vegter, and A. van den Boogaard, International Journal of Plasticity 22, 557-580 (2006).

17. A. van den Boogaard, and J. Huétink, Computer Methods in Applied Mechanics and Engineering 195, 6691-6709 (2006). 